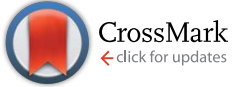

Cite this: RSC Adv., 2017, 7, 2074

Received 12th November 2016 Accepted 5th December 2016

DOI: 10.1039/c6ra26703c

www.rsc.org/advances

\section{Broadband localized electric field enhancement produced by a single-element plasmonic nanoantenna}

\author{
Zhengdong Yong, ${ }^{a}$ Chensheng Gong, ${ }^{a}$ Yongjiang Dong, ${ }^{a}$ Senlin Zhang ${ }^{a}$ \\ and Sailing $\mathrm{He}^{\star \mathrm{ab}}$
}

We propose a novel design of a broadband plasmonic nanoantenna, investigate it numerically using finitedifference time-domain methods, and explain its performance using the analysis of charge distribution in addition to a multipole expansion. The custom-designed single-element nanoantenna consists of a modified gold ring structure with a bowtie-shaped spike inside. In contrast to the spectral response of extinction, the broadband localized electric field intensity enhancement is achieved over a bandwidth of $850 \mathrm{~nm}$ in the near infrared spectrum. Up to 26- and 22-fold field enhancements near the bowtie spike are obtained the peak and even in the dip region of the extinction spectrum, respectively. Moreover, the nanostructure exhibits high tunability of its spectral features by modifying the structural parameters. We further demonstrate that the proposed nanoantenna can provide broadband spontaneous emission rates and quantum efficiency enhancements when a low-quantum efficiency emitter is introduced.

\section{Introduction}

Inspired by radio and microwave antennas, optical antennas operating at visible and near-infrared frequencies have emerged as a powerful tool in nano-optics, as they can efficiently link free-propagating light with localized fields at the nanometer scale. ${ }^{1,2}$ Due to the localized surface plasmon resonances arising from the collective oscillation of free electrons in metals, optical antennas allow for large electromagnetic field enhancement, deep subwavelength confinement of light, and tailoring of the local density of photonic states. ${ }^{3,4}$ These promising abilities have ignited the development of a vast variety of applications, including surface-enhanced Raman scattering spectroscopy, ${ }^{5,6}$ fluorescence enhancement, ${ }^{7,8}$ optical manipulation ${ }^{9,10}$ and nonlinearity. ${ }^{11,12}$ As the resonance decisively depends on the shape, dimensions, and material of the antenna, a large variety of plasmonic antenna structures have been proposed, such as nanorods, ${ }^{13}$ bow-ties ${ }^{14}$ nanorings, ${ }^{15}$ and Yagi-Uda antennae. ${ }^{16}$ Though all of these antennas have demonstrated the ability to confine light in the nanoscale and strongly enhance local fields, their narrow-band spectra with sharp resonances are a major challenge for applications that require devices operating over a wide range of frequencies. For example, antennas operating over a wide frequency spectrum are essential to improve energy

${ }^{a}$ Centre for Optical and Electromagnetic Research, State Key Laboratory of Modern Optical Instrumentations, Zhejiang University, Hangzhou 310058, China. E-mail: sailing@kth.se

${ }^{b}$ Department of Electromagnetic Engineering, School of Electrical Engineering, Royal Institute of Technology (KTH), S-100 44 Stockholm, Sweden harvesting efficiency of photovoltaic devices. ${ }^{17,18}$ Broadband optical antennas are also highly desirable for surface enhanced Raman scattering, fluorescence enhancement, and higher harmonic generation, which are intrinsically multi-wavelength and broadband in nature. ${ }^{6,19,20}$

To surpass the inherently limited narrow-band operation of infrared frequencies, several topologies have been explored, such as antennas with long aspect radio, ${ }^{21,22}$ fractal geometry, ${ }^{23,24}$ multiple-segment arrangements, ${ }^{25,26}$ wavelength-size 2D structures ${ }^{27}$ and log-periodic antennas..$^{20,28,29}$ However, they all have some limitations; for instance, the locations of hot spots in fractal antennas ${ }^{23}$ are wavelength-dependent, the bandwidth of multiple dipole antennas is constrained to the number of resonant elements, ${ }^{25}$ and photonic-plasmonic antennas require particle arrays of multiple periods, leading to impractically large footprints. ${ }^{26}$ Recently, analogous to the microwave designs of broadband antennas, trapezoidal logperiodic antennas have been proposed and used for multispectral applications. ${ }^{20,30}$ Coupled antennas are also good candidates for broadband operation..$^{31,32}$ However, all the nanoantenna designs in literature require at least two separated elements. These disconnected structures can only be fabricated on substrates, which hampers their biochemistry applications in aqueous solutions or in vivo. Though the coupled antennas provide a much higher field intensity, for some practical applications (i.e., fluorescence enhancement of fluorophores), high excitation intensities are often detrimental to their efficiency and photostability. ${ }^{33}$ Furthermore, the small sizes of the hot spots of coupled antennas limit the interaction to just a few emitters and prevent the development of large-area chips. For 
these reasons, it is of great significance and remains a great challenge to design a single-element broadband plasmonic nanoantenna with larger hot spot areas.

In this paper, we have proposed a single-element modified ring nanostructure which provides broadband localized field enhancement in the same spatial position. The spectral responses of the nanoantenna in terms of cross sections and field enhancement near the tip are first studied. Then, the analysis of charge distributions and multipole expansions are performed to investigate the underlying physics. Finally, broadband spontaneous emission rates and quantum efficiency enhancements are demonstrated when a quantum emitter is placed inside the nanoantenna. Such single-element broadband antennas will hold great potential in many applications, such as nanolasers, biosensors, surface enhanced Raman scattering, and nonlinear optics.

\section{Methods}

Three-dimensional finite-difference time-domain calculations were performed using commercially available software package (Lumerical FDTD Solutions Inc. v8.6) to investigate the optical properties of the antenna. Fig. 1(a) shows the schematic of our proposed single-element broadband plasmonic nanostructure. It is represented by a modified gold ring resonator with a bowtieshaped spike inside. The relevant structural parameters are the bowtie's length $L$, opening angle $\theta$, the inner radius $R_{\mathrm{in}}$, and the outer radius $R_{\text {out }}$ of the ring. Considering the fabrication limits, and in order to avoid sharp edges that are difficult to fabricate, we model the bowtie's tip as rounded with a radius of curvature of $10 \mathrm{~nm}$. In the following, the typical geometrical parameters are chosen; namely, $L=110 \mathrm{~nm}, \theta=50^{\circ}, R_{\text {in }}=110 \mathrm{~nm}, R_{\text {out }}=$ $180 \mathrm{~nm}$, and the height $T$ of the antenna is $40 \mathrm{~nm}$.

To first study the optical properties of the single antenna, a total-field scattered-field (TFSF) source polarized along the $x$ direction was normally-incident to it, as shown in Fig. 1(a). Perfectly matched layers were employed in every face of the simulation box. Mesh override regions covering different volumes of the structure were used for fine resolution of the structure. Specifically, a mesh size of $0.5 \mathrm{~nm}$ was used near the bowtie's tip and $1 \mathrm{~nm}$ for other regions. Near field distributions were recorded by field monitors, and the extinction cross section was calculated by the sum of the scattering and absorption cross sections. ${ }^{3}$ Then, for the simulation of the emission process modified by the nanoantenna, a point dipole source oriented in the $x$-direction (considered as an isolated emitter) is placed inside the nanoantenna. The radiated emitted power $P_{\text {rad }}$ and the total emitted power $P_{\text {tot }}$ in the presence of the antenna were calculated by integrating the power flow over a closed surface surrounding the dipole source at respective distances of $1 \mu \mathrm{m}$ and $5 \mathrm{~nm}$. The total and radiative decay rates enhancement $\left(\Gamma_{\mathrm{rad}}=P_{\mathrm{rad}} / P_{0}\right.$ and $\left.\Gamma_{\text {tot }}=P_{\text {tot }} / P_{0}\right)$ can then be obtained by normalizing the emitted power with the radiated emitted power $P_{0}$ of the same dipole source in the absence of the antenna. ${ }^{4}$

Finally, to further understand the origin of the spectral response of the nanoantenna, we perform a multipole expansion $^{34}$ of the total scattering response of the nanoantenna for plane wave excitation, according to

$$
C_{\text {scat }}=\frac{\pi}{k^{2}} \sum_{l=1}^{\infty} \sum_{m=-l}^{l}(2 l+1)\left[\left|a_{l m}\right|^{2}+\left|b_{l m}\right|^{2}\right]
$$

here, $a_{l m}$ and $b_{l m}$ are the multipole coefficients of electric and magnetic modes, respectively. Using the reciprocity theorem, ${ }^{2}$ we also consider the same dipole excitation scenario as before to investigate the near field of the nanoantenna. For all calculations, the optical constant of the gold is modeled with a Drude-Lorentz dispersion model, ${ }^{35,36}$ and we assume that the whole structure is immersed in water $(\varepsilon=1.77)$.

\section{Results and discussion}

The near field intensity enhancements with respect to the corresponding incident field at the two monitoring points $P_{1}$ and $P_{2}$ (5 nm distant from the tip and ring marked in Fig. 1) and the extinction cross-sections of the single nanoantenna are plotted in Fig. 1(b). It is evident that our plasmonic nanoantenna has two pronounced extinction peaks around $\lambda_{1}=1100 \mathrm{~nm}$ and $\lambda_{2}=$ $1700 \mathrm{~nm}$. A lower peak around $\lambda_{3}=850 \mathrm{~nm}$ is also presented in both the extinction and near field intensity spectrum. Most interestingly, in contrast to the extinction characteristics, we observe a relatively large field intensity enhancement at $P_{1}$ over a broad spectral range, and the full-width-half-maximum (FWHM) bandwidth is about $850 \mathrm{~nm}$. Up to 26- and 22-fold field enhancements near the bowtie spike are obtained at the peak and even in the dip region of the extinction spectrum, respectively. In addition, this broadband behavior can only be observed inside the modified ring. To understand the operating principle of the nanoantenna, the charge distribution analysis of the plasmonic modes at specific wavelengths are performed. A simple way of visualizing the charges is to calculate the normal component of the electric field $E_{z}$ on a plane $2 \mathrm{~nm}$ above the structure since the field component is not contained in the illumination and orientation is directly related to the sign of the charges. ${ }^{37}$ As clearly shown in Fig. 1(c), the related mode profile at $\lambda_{3}$ can be assigned to a quadrupole-like oscillation. Due to the symmetry breaking, induced by the additional bowtie spike, this usual dark mode can be directly excited by normal incidence. With regard to the resonance around $\lambda_{1}$ shown in Fig. 1(i), the strong field is mainly concentrated around the bowtie spike. From Fig. 1(f) and (k), a typical symmetric mode of the modified ring around the resonance $\lambda_{2}$ and strong field around the ring can be observed. ${ }^{15}$ Particularly, for the electron oscillations corresponding to the dip of the extinction spectrum in Fig. 1(e), the total dipole moment (observed at the far field) is much smaller, but a large near field enhancement inside the nanoantenna can still be produced as shown in Fig. 1(j) and the charges are mainly localized near the bowtie tips. These charge distribution analysis can provide us with an intuitive understanding of the plasmonic modes of the proposed nanoantenna. The additional bowtie spike inside the nanoring serves as an electron reservoir and enables the focalization of electric fields at its apex due to the lightning rod effect. ${ }^{38,39}$ To further 
<smiles>[3H][Mg][Mg]</smiles>
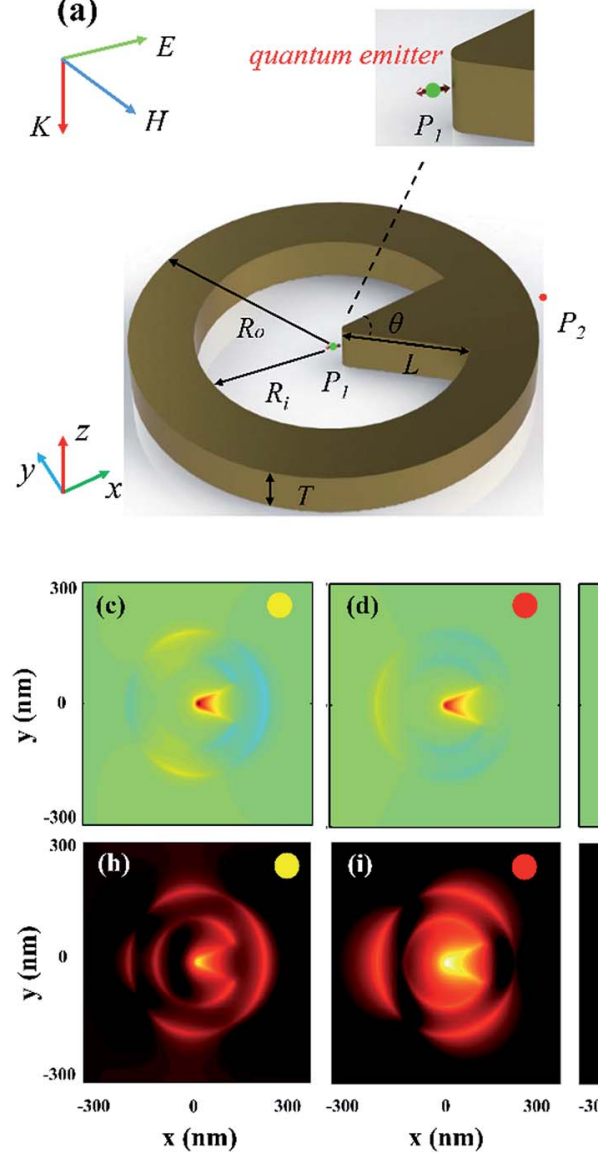

(b)

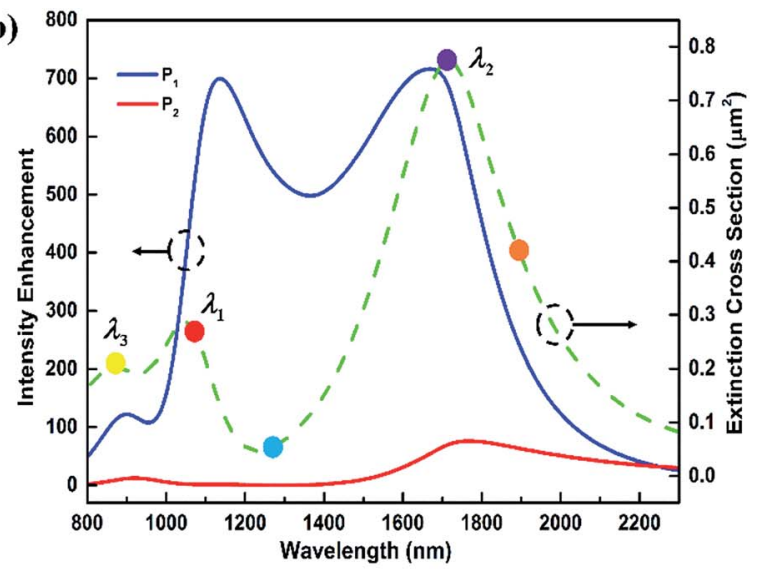

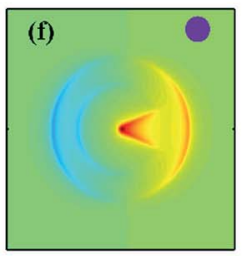
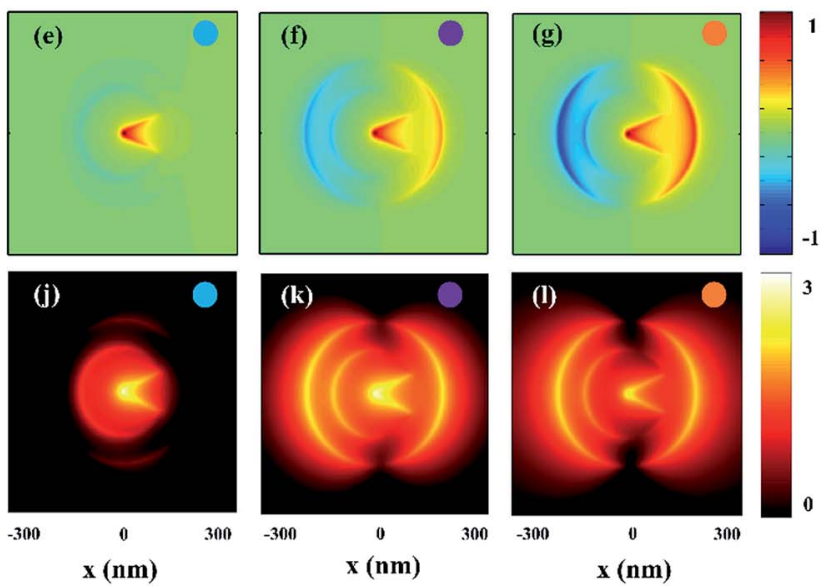

Fig. 1 (a) Schematic illustration of a single-element broadband plasmonic nanoantenna. It is represented by a modified gold ring resonator with a bowtie-shaped spike inside. The whole structure is immersed in water. (b) FDTD simulations of the near field intensity enhancement at $P_{1}$ (blue solid), $P_{2}$ (red solid) and extinction cross section (green dashed). (c-g) Real part of the normal component of the electric field $2 \mathrm{~nm}$ above the structure at the selected wavelengths shown in panel $b$. The sign of the field gives the charge distribution of the plasmonic modes supported by the structure. $(\mathrm{h}-\mathrm{l})$ Near-field intensity in logarithmic scale plotted on the same plane cutting the structure as for the cases shown in panels (c-g). The typical geometrical parameters are chosen, namely, $L=110 \mathrm{~nm}, \theta=50^{\circ}, R_{\text {in }}=110 \mathrm{~nm}, R_{\text {out }}=180 \mathrm{~nm}$, and the height $T$ of the antenna is $40 \mathrm{~nm}$.

evaluate the performance of the proposed antenna, we also compare it to the isolated nano-triangle with the same dimensions as the one protruding inside the ring and the popular bowtie design for a wavelength of $1100 \mathrm{~nm}$. The isolated nanotriangle has a resonant peak around $800 \mathrm{~nm}$ in Fig. 2(a), with FWHW about $100 \mathrm{~nm}$, which is much narrower than our proposed structure. Thus the nanoring plays a critical role in this broadband antenna. Fig. 2(b) also shows the spectral response of bowtie antennas. ${ }^{14}$ We find a 40 -fold electric field enhancement at resonance with a bandwidth of $300 \mathrm{~nm}$.

In order to further investigate the origin of the spectral response of our antennas, a multipole analysis is performed for both plane wave and dipole excitation. The results of the nonnegligible coefficients for plane waves are presented in Fig. 3(a). The $a_{2}$ coefficient, corresponding to an electric quadrupole contribution, shows a resonant behavior around the operation wavelength $\lambda_{3}$. This is consistent with the charge distribution analysis in Fig. 1(c). Furthermore, the two resonant peaks of the extinction spectrum around $\lambda_{1}$ and $\lambda_{2}$ mainly originate from the electric dipole contribution. The small electric dipole moment between these two resonances results in the dip in the extinction spectrum. In fact, we can comprehend the interaction between the designed structure and light wave by considering the antenna as simply two coupled dipolar resonators. Their mode profiles on resonance are shown in Fig. 1(d) and (f). We note again here that a dipolar resonance is characterized by a phase that matches the phase of the incident field on one side of the resonance and is out-of-phase on the other side. In our case, where the two dipolar resonances have different resonant frequencies, there will be some regions of the spectrum where they are in phase or out of phase, forming a higher radiant mode (constructive interference) or a lower radiant mode (destructive interference). The interaction between two bright dipolar modes has recently been studied in the far field region, and the destructive interference gives rise to the Fano resonance. ${ }^{40}$ However, interference of the incident and scattered light waves generates a more complex near-field pattern. In particular, it is the near field enhancement and not the extinction properties of the antenna that influences the efficiency of SERS or fluorescence. We may qualitatively 
(a)

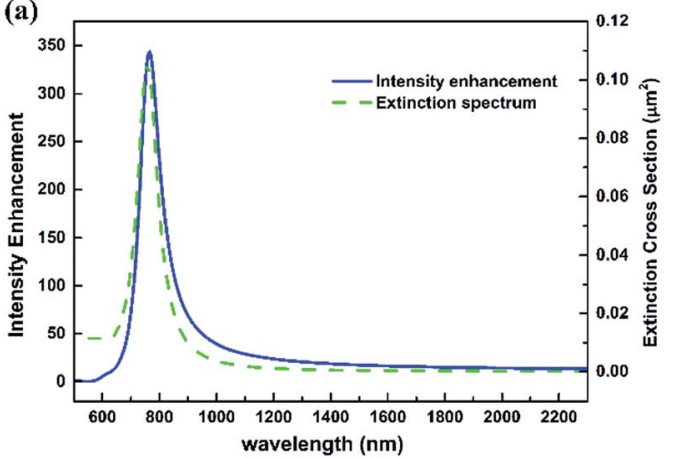

(b)

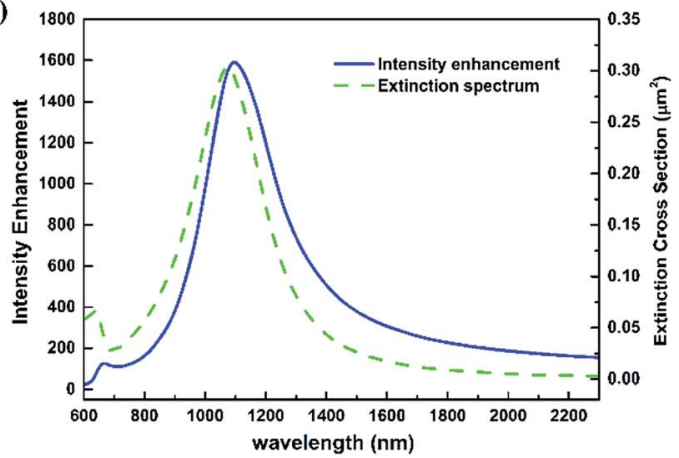

Fig. 2 (a) FDTD simulations of the near field intensity enhancement at the point $5 \mathrm{~nm}$ distant from the bowtie tip (blue solid), and extinction cross section (green dashed) for an isolated nanotriangle with the same dimensions as the one protruding inside the ring. (b) FDTD simulations of the near field intensity enhancement at the point $5 \mathrm{~nm}$ distant from the bowtie tip (blue solid), and extinction cross section (green dashed) of bowtie antennas for a resonant wavelength of $1100 \mathrm{~nm}$, the gap of the bowtie antennas is $20 \mathrm{~nm}$.

attribute the broadband localized field enhancement to the near field constructive interference of the two dipolar resonances. To evaluate it more quantitatively, due to the reciprocity theorem, ${ }^{2}$ we calculate the normalized decay rates for an on-axis $x$-oriented dipole located at $P_{1}$ and use the same multipole expansion of the radiated field as before. ${ }^{41}$ An expected broadband radiative decay rate is shown in Fig. 3(b). More importantly, we emphasize that the dominant contribution of the electric dipole to the radiative decay rate is broadband for dipole excitation in Fig. 3(c), in contrast to that of plane wave excitation. By using multipole expansion, it aptly explains the different spectral responses between the far field and the near field.

As mentioned above, the structural parameters of the nanoantenna are critical for its optical properties. Some parametric studies are performed to give us insights as to how they affect the performance of the nanoantenna. Fig. 4(a) and (b) illustrate the near field intensity spectrum at $P_{1}$ when $L$ and $R_{\text {out }}$ are changed, respectively, while other parameters are kept the same. More than one order of localized electric field enhancement over a broadband spectral range, up to $1000 \mathrm{~nm}$, can be observed in all the studies. From Fig. 4(a), one sees an obvious (a)

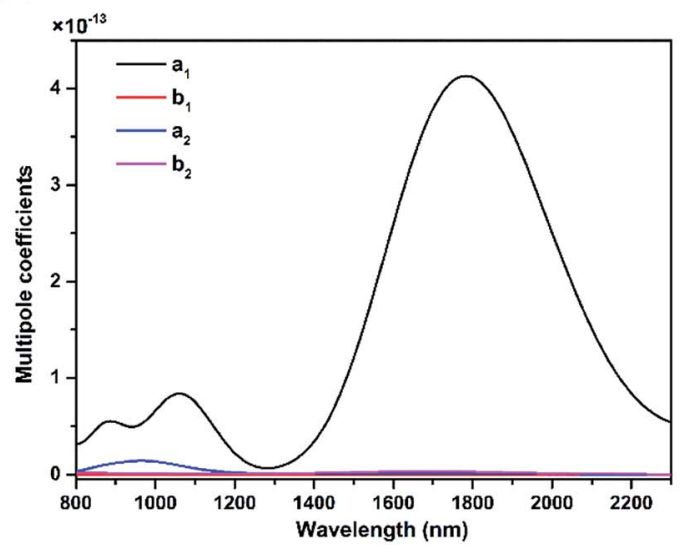

(b)

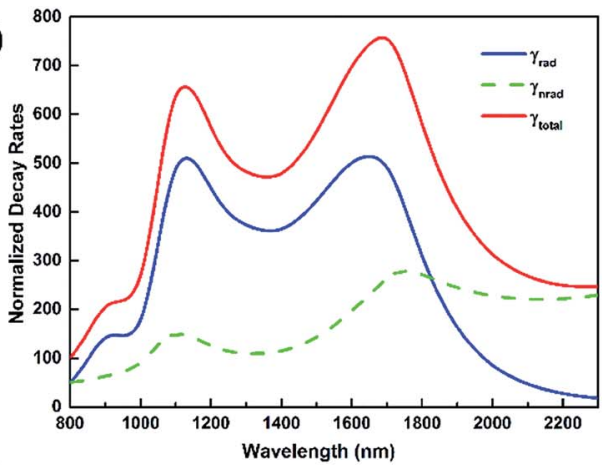

(c)

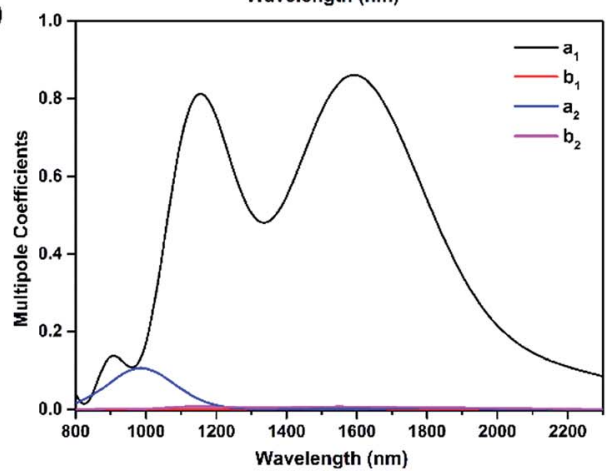

Fig. 3 (a) Multipole expansion of the total scattered light by a single nanostructure under plane wave excitation. Only the non-negligible multipole coefficients are shown. (b) Normalized decay rates spectrum for an on-axis dipole oriented parallel to the bowtie spike and located at $P_{1}$. The blue solid, green dashed, and red solid curves are for the radiative, nonradiative and total decay rates, respectively. (c) Multipole expansion of the total radiated power by the same nanostructure under dipole excitation. 

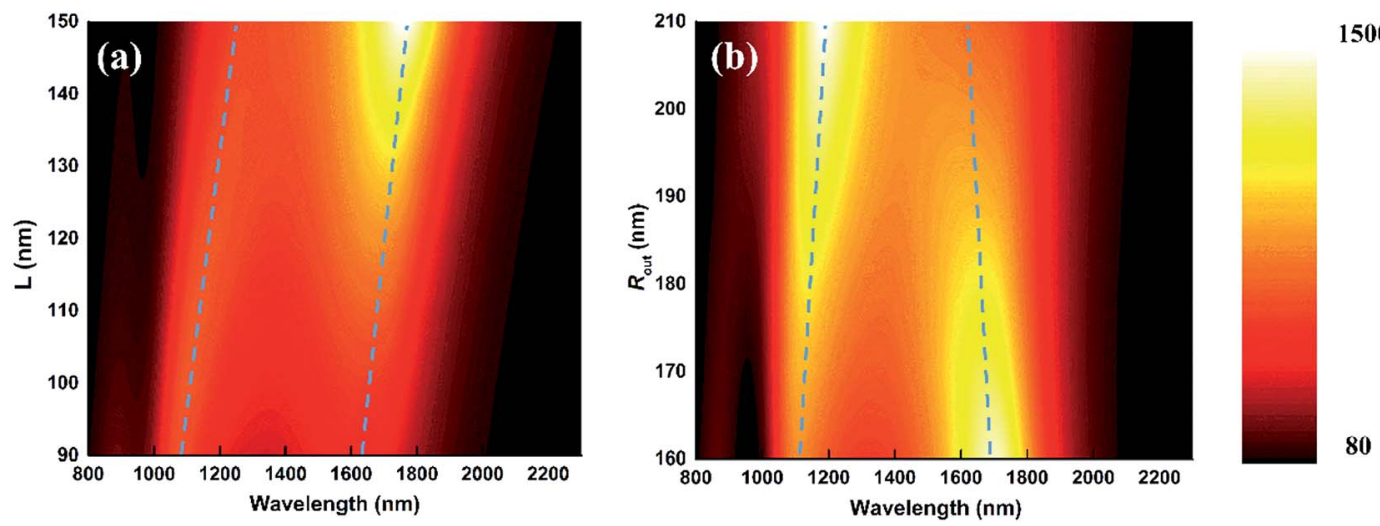

Fig. 4 Simulated near field intensity enhancement spectra at $P_{1}$ as the length $L$ of the bowtie spike (a) and outer radius $R_{\text {out }}$ of ring (b) vary, with other parameters kept the same. The dashed lines correspond to the peaks of near field intensity.

red-shift of the two resonant peaks and an increasing intensity enhancement of the long wavelength resonance as the length $L$ increases. While in Fig. 4(b), as $R_{\text {out }}$ increases from $160 \mathrm{~nm}$ to $210 \mathrm{~nm}$, the two pronounced resonances of the near field intensity enhancement gradually contract to the center wavelength, with the strength at the short wavelength increases and the other decreases. This is due to a decreasing coupling strength between the inner and outer surfaces of the nanoring. ${ }^{15}$ Therefore, both the length $L$ of the bowtie spike and the outer radius $R_{\text {out }}$ of the ring need to be carefully designed to engineer the near field of the nanoantenna.

Inspired by the broadband localized field enhancement in the modified ring nanoantenna, we propose its potential application for controlling the radiative properties of quantum emitters. As was first studied by Purcell, the radiation dynamic of quantum emitters can be modified by the local density of the optical states. ${ }^{4,42,43} \mathrm{~A}$ representative broadband and large Purcell

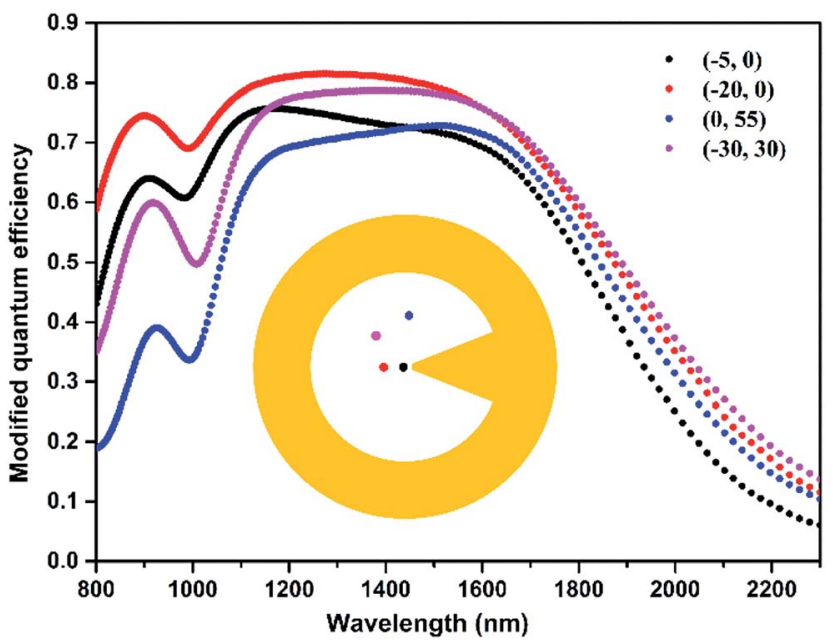

Fig. 5 The calculated modified quantum efficiency of a quantum emitter located at different positions inside the proposed nanoantenna (shown in the inset). We set the tip of the bowtie spike as the origin of the coordinates, and the intrinsic quantum efficiency $\eta_{0}$ of the quantum emitter is 0.25 . enhancement with a bandwidth of $800 \mathrm{~nm}$ is shown in Fig. 3(b), which has never been achieved by a single-element nanostructure so far. In the spectral range of interest, the nonradiative decay rates due to metal loss are much smaller than the radiative rates, which result in a high quantum efficiency. As the total fluorescence enhancement is a product of field enhancement factor in the excitation process and quantum efficiency enhancement in the emission process, it is necessary to study the modified quantum efficiency of a quantum emitter by our proposed broadband nanoantenna. Previous studies have demonstrated that low-quantum efficiency emitters have much higher fluorescence enhancement than high-quantum efficiency emitters because their intrinsic quantum efficiency has a greater potential to be improved by the presence of the nanoantenna. ${ }^{44}$ In our studies, we set the tip of the bowtie spike as the origin of the coordinates and assume a quantum emitter located at different positions inside of the typical nanoantenna with intrinsic quantum efficiency $\eta_{0}=0.25$. The modified quantum efficiency $\eta$ related to $\eta_{0}$ is calculated according to $\eta=\frac{\eta_{0} \cdot \Gamma_{\text {rad }}}{1-\eta_{0}+\eta_{0} \cdot \Gamma_{\text {tot }}}$, and shown in Fig. 5. It is obvious that the modified quantum efficiency of a quantum emitter is highly improved by the nanoantenna, and all the curves depict a broadband quantum efficiency enhancement. It indicates that the structure has a large active volume, allowing for collective coupling of many quantum emitters in a reproducible and predictable manner. Compared to the traditional nanoring structure, ${ }^{45}$ the additional bowtie spike inside can broaden the spectral response of the near field and provides a much larger intensity enhancement due to a "lightning rod effect". As a result, the proposed single-element nanoantenna is very promising for practical applications.

\section{Conclusions}

In summary, we have presented and demonstrated by employing FDTD calculations an efficient strategy to engineer broadband localized field enhancement in the same spatial area. Specifically, by introducing a bowtie spike inside the nanoring, the single-element nanoantenna shows a large near field 
intensity enhancement for an $850 \mathrm{~nm}$ bandwidth in the nearinfrared spectrum. Up to 26- and 22-fold field enhancements near the bowtie spike are obtained at the peak and even in the dip region, respectively, of the extinction spectrum. The analysis of the charge distribution and multipole expansion clearly reveal the origin of the spectral response of the nanoantenna. By identifying its multipolar nature, the dominant electric dipolar contributions give rise to the different spectral responses between the near field and the far field. In addition, the nanostructure exhibits high tunability of its spectral features by modifying geometrical parameters. From the application point of view, the proposed nanoantenna has great potential in controlling the radiative properties of a quantum emitter. The strong field enhancement inside the nanoantenna simultaneously enables high Purcell and broadband quantum efficiency enhancement of quantum emitters, outperforming that of traditional plasmonic rings and dimmer antennas. These finding provides a new design strategy for broadband localized field enhancement, such as the design of the same ring but with two bowtie spikes facing each other. It could be exploited for a range of other applications, such as harmonic generation and nanolasers. ${ }^{46}$

\section{Acknowledgements}

This work was partially supported by the National Natural Science Foundation of China (No. 91233208 and 61178062), the National High Technology Research and Development Program (863 Program) of China (No. 2013AA014401), the Program of Zhejiang Leading Team of Science and Technology Innovation, and Swedish VR grant (No. 621-2011-4620).

\section{Notes and references}

1 L. Novotny and N. van Hulst, Nat. Photonics, 2011, 5, 83-90.

2 P. Biagioni, J. S. Huang and B. Hecht, Rep. Prog. Phys., 2012, 75, 024402.

3 S. A. Maier, Plasmonics: fundamentals and applications, Springer, 2007.

4 L. Novotny and B. Hecht, Principles of nano-optics, Cambridge University Press, 2006.

5 J. Xie, Z. Q. Zhang, J. Y. Lee and D. I. C. Wang, ACS Nano, 2008, 2, 2473-2480.

6 Y. Chu, M. G. Banaee and K. B. Crozier, ACS Nano, 2010, 4, 2804-2810.

7 P. Anger, P. Bharadwaj and L. Novotny, Phys. Rev. Lett., 2006, 96, 113002.

8 A. Kinkhabwala, Z. Yu, S. Fan, Y. Avlasevich, K. Müllen and W. E. Moerner, Nat. Photonics, 2009, 3, 654-657.

9 W. Zhang, L. Huang, C. Santschi and O. J. F. Martin, Nano Lett., 2010, 10, 1006-1011.

10 K. Wang, E. Schonbrun, P. Steinvurzel and K. B. Crozier, Nat. Commun., 2011, 2, 469.

11 K. Thyagarajan, S. Rivier, A. Lovera and O. J. F. Martin, Opt. Express, 2012, 20, 12860-12865.

12 H. Aouani, M. Rahmani, M. Navarro-Cía and S. A. Maier, Nat. Nanotechnol., 2014, 9, 290-294.
13 P. Mühlschlegel, H.-J. Eisler, O. J. F. Martin, B. Hecht and D. W. Pohl, Science, 2005, 308, 1607-1609.

14 P. J. Schuck, D. P. Fromm, A. Sundaramurthy, G. S. Kino and W. E. Moerner, Phys. Rev. Lett., 2005, 94, 017402.

15 J. Aizpurua, P. Hanarp, D. S. Sutherland, M. Käll, G. W. Bryant and F. J. García de Abajo, Phys. Rev. Lett., 2003, 90, 057401.

16 T. H. Taminiau, F. D. Stefani and N. F. van Hulst, Opt. Express, 2008, 16, 10858-10866.

17 M. A. Green and S. Pillai, Nat. Photonics, 2012, 6, 130.

18 H. A. Atwater and A. Polman, Nat. Mater., 2010, 9, 205-213. 19 G. Lu, J. Liu, T. Zhang, H. Shen, P. Perriat, M. Martini, O. Tillement, Y. Gu, Y. He, Y. Wang and Q. Gong, Nanoscale, 2013, 5, 6545-6551.

20 M. Navarro-Cia and S. A. Maier, ACS Nano, 2012, 6, 35373544.

21 J. Dorfmuller, R. Vogelgesang, W. Khunsin, C. Rockstuhl, C. Etrich and K. Kern, Nano Lett., 2010, 10, 3596-3603.

22 B. N. Khlebtsov and N. G. Khlebtsov, J. Phys. Chem. C, 2007, 111, 11516-11527.

23 G. Volpe, G. Volpe and R. Quidant, Opt. Express, 2011, 19, 3612-3618.

24 R. U. Tok and K. Şendur, Opt. Lett., 2014, 39, 6977-6980.

25 E. S. Ünlü, R. U. Tok and K. Șendur, Opt. Express, 2011, 19, 1000-1006.

26 R. Blanchard, S. V. Boriskina, P. Genevet, M. A. Kats and F. Capasso, Opt. Express, 2011, 19, 22113-22124.

27 C. Kan, X. Zhu and G. Wang, J. Phys. Chem. B, 2006, 110, 4651-4656.

28 R. S. Pavlov, A. G. Curto and N. F. van Hulst, Opt. Commun., 2012, 285, 3334-3340.

29 J. Yang, F. Kong, K. Li and S. Sheng, Opt. Commun., 2015, 342, 230-237.

30 H. Aouani, M. Rahmani, H. Šípová, V. Torres, K. Hegnerová, M. Beruete and S. A. Maier, J. Phys. Chem. C, 2013, 117, 18620-18626.

31 R. Zhou, J. Ding, B. Arigong, Y. Lin and H. Zhang, J. Appl. Phys., 2013, 114, 184305.

32 Z. D. Yong, S. Zhang, Y. Dong and S. He, Prog. Electromagn. Res., 2015, 153, 123-131.

33 K. M. Dyumaev, A. A. Manenkov, A. P. Maslyukov, G. A. Matyushin, V. S. Nechitailo and A. M. Prokhorov, J. Opt. Soc. Am. B, 1992, 9, 143-151.

34 P. Grahn, A. Shevchenko and M. Kaivola, New J. Phys., 2012, 14, 093033.

35 A. Vial, A.-S. Grimault, D. Macias, D. Barchiesi and M. L. de la Chapelle, Phys. Rev. B: Condens. Matter Mater. Phys., 2005, 71, 085416.

36 P. B. Johnson and R. W. Christy, Phys. Rev. B: Solid State, 1972, 6, 4370-4379.

37 C. Rockstuhl, F. Lederer, C. Etrich, T. Zentgraf, J. Kuhl and H. Giessen, Opt. Express, 2006, 14, 8827-8836.

38 D. Wang, T. Yang and K. B. Crozier, Opt. Express, 2010, 18, 10388-10394.

39 A. Li and S. Li, Nanoscale, 2014, 6, 12921-12928.

40 A. Lovera, B. Gallinet, P. Nordlander and O. J. Martin, ACS Nano, 2013, 7, 4527-4536. 
41 E. Rusak, I. Staude, M. Decker, J. Sautter, A. E. Miroshnichenko, D. A. Powell and Y. S. Kivshar, Appl. Phys. Lett., 2014, 105, 221109.

42 E. M. Purcell, Phys. Rev., 1946, 69, 681.

43 P. Fauché, S. Ungureanu, B. Kolaric and R. A. Vallée, J. Mater. Chem. C, 2014, 2, 10362-10368.
44 L. Rogobete, F. Kaminski, M. Agio and V. Sandoghdar, Opt. Lett., 2007, 32, 1623-1625.

45 A. Rakovich, P. Albella and S. A. Maier, ACS Nano, 2015, 9, 2648-2658.

46 M. A. Noginov, G. Zhu, A. M. Belgrave, R. Bakker, V. M. Shalaev, E. E. Narimanov and U. Wiesner, Nature, 2009, 460, 1110-1112. 